\title{
Kinetic Theory of Subsonic and Supersonic Transport Processes for Screened Coulomb Interactions
}

\author{
H. E. WILHELM \\ Colorado State University, Fort Collins, Colorado 80521 \\ (Z. Naturforsch. 25 a, 322-328 [1970]; received 7 November 1969)
}

\begin{abstract}
A statistical theory of the intercomponent transport processes is given for nonisothermal plasmas consisting of electron and ion components, when the particles interact through Coulomb collisions screened electrostatically beyond the Debye-radius. The associated collision integrals are evaluated analytically for arbitrary drift velocities of the components, and under consideration of the velocity dependence of the Coulomb logarithm. The resultant analytical relations form consistent mathematical foundations for a transport theory of systems with collisional Coulomb interactions. It is shown that different properties of the transport processes and the effective Coulomb logarithms result for subsonic and supersonic drift velocities of the components.
\end{abstract}

\section{Introduction}

A kinetic investigation starting from the Boltzmann equation may be based on various integration formalisms ${ }^{*}, 1$. A physically illustrative and mathematically elegant method is the expansion of the distribution function around a local equilibrium distribution function in terms of Hermite polynomials, the expansion coefficients being given as moments of the distribution function ${ }^{2}$. In restricting this orthogonal development to the first thirteen moments, which have a simple physical meaning, the velocity distribution of the $r$-th particle component is ${ }^{3}$ :

$$
\begin{aligned}
& f_{r}=f_{r}^{(0)}\left[1+\frac{m_{r}}{2 k T_{r}}\left(\frac{\overleftrightarrow{\boldsymbol{p}}_{r}}{p_{r}}-\overleftrightarrow{\boldsymbol{\delta}}\right): \boldsymbol{c}_{r} \boldsymbol{c}_{r}\right. \\
& \left.+\frac{m_{r}}{p_{r} k T_{r}}\left(\frac{m_{r} c_{r}^{2}}{5 k T_{r}}-1\right) \boldsymbol{q}_{r} \cdot \boldsymbol{c}_{r}\right], \\
& \boldsymbol{c}_{r} \equiv \boldsymbol{v}_{r}-\left\langle\boldsymbol{v}_{r}\right\rangle \text {. }
\end{aligned}
$$

The perturbations of the distribution function are caused by different types of transport processes: i) the viscous stresses $\pi_{r, i j}$ and the heat currents $q_{r, i}$, i. e. transport processes due mainly to the in-

Reprints request to Dr. H. E. Wilhelm, Department of Mechanical Engineering, Colorado State University, Fort Collins, Colorado 8052, USA.

* Standard notations are used, e. g., $m_{r s}=m_{r} m_{s} /\left(m_{r}+m_{s}\right)$ $=$ reduced mass, $T_{r s}=m_{r s}\left[\left(T_{r} / m_{r}\right)+\left(T_{s} / m_{s}\right)\right]$, etc.

${ }^{1}$ H. Grad, Principles of the Kinetic Theory of Gases, Encyclopedia of Physics, Vol. XII, Springer-Verlag, New York 1958.

2 H. Grad. Comm. Pure Appl. Math. 2, 331 [1949a.

${ }^{3}$ H. Grad, Comm. Pure Appl. Math. 5, 257 [1952].

4 V. M. ZHDANOv, PMM (USSR) 26, 280 [1962].

5 R. S. Cohen, L. Spitzer, Jr., and P. McRoutly, Phys. Rev. 80, 230 [1950]. homogeneities in the component, and ii) the momentum and energy exchange between different components due to intercomponent nonuniformities. Both types of transport processes influence each other to an extent determined by the nonuniformities in $\left(\nabla n_{r}, \nabla T_{r}, \nabla\left\langle\boldsymbol{v}_{r}\right\rangle, \ldots\right)$ and between $\left(T_{r}-T_{s} \neq 0\right.$, $\left.\left\langle\boldsymbol{v}_{r}\right\rangle-\left\langle\boldsymbol{v}_{s}\right\rangle \neq \mathbf{0}, \ldots\right)$ the components ${ }^{4}$.

The theory of transport processes for plasmas is in part incomplete and phenomenological. Except for special applications, e. g. the relaxation of test particles, it is commonly implied that the baro-drift velocities are infrasonic ${ }^{4-12}$. Another standard procedure is the integration of the collision integrals after replacing the velocity dependent Coulomb logarithm by a thermal average ${ }^{4-12}$. This average Coulomb logarithm is assumed to be the same for the different transport processes ${ }^{4-13}$. Further, identical average Coulomb logarithm are used for subsonic and supersonic processes ${ }^{11,12}$, respectively, i. e. for

$$
\frac{1}{2} m_{r s}\left(\left\langle\boldsymbol{v}_{s}\right\rangle-\left\langle\boldsymbol{v}_{r}\right\rangle\right)^{2} \lessgtr k T_{r s} .
$$

For these reasons, it seems desirable to develop more consistent theoretical foundations for the plasma transport processes resulting from collisional

6 L. D. Landau, Phys. Z. USSR 10, 154 [1936].

7 S. Gvozdover, Phys. Z. USSR 12, 164 [1937].

8 T. G. Cowling, Proc. Roy. Soc. London A 183, 453 [1945].

9 J. H. CAHn, Phys. Rev. 75, 293, 346, 838 [1953].

10 S. I. Braginskit, Sov. Phys. -JETP 6, 358 [1958].

11 R. Herdan and B. S. Liley, Revs. mod. Phys. 32, 731 [1960].

12 L. SpITzer, Jr., Physics of Fully Ionized Gases, Interscience, New York 1966.

13 Note also that different authors ${ }^{4-12}$ assume in part different average Coulomb logarithms. 
Coulomb interactions. To this end, the intercomponent momentum and energy transport is analyzed for quasi-homogeneous, nonisothermal plasmas without noticeable viscous stresses and heat currents, $\pi_{r, i j} \rightarrow 0$ and $q_{r, i} \rightarrow 0$. The collision integrals are evaluated analytically under inclusion of the velo. city dependent Coulomb logarithm. Further, consideration is given not only to subsonic but also to supersonic transport conditions.

\section{Theoretical Principles}

It is well established that the collisional Coulomb interactions in a rarefied plasma can be treated within the frame of the Boltzmann equation, or its Fokker-Planck expansion for successive gentle interactions ${ }^{5}$. In order to include close and distant collisions, the kinetic considerations will be based on the Boltzmann collision integral.

\section{Kinetic Equation}

The change of the distribution function $f_{r}\left(\boldsymbol{v}_{r}, \boldsymbol{r}, t\right)$ of the particle velocities $\boldsymbol{v}_{r}$ of an arbitrary component $(r)$ in the vicinity of a point $\boldsymbol{r}$ is described by the kinetic equation ${ }^{14}$ :

$\frac{\partial}{\partial t} f_{r}+\frac{\partial}{\partial \boldsymbol{r}} \cdot\left(\boldsymbol{v}_{r} f_{r}\right)+\frac{\partial}{\partial \boldsymbol{v}_{r}} \cdot\left(\boldsymbol{w}_{r} f_{r}\right)=\sum_{s} C_{r s}$,

which expresses the continuity of $f_{r}\left(\boldsymbol{v}_{r}, \boldsymbol{r}, t\right)$ in presence of exterior particle accelerations $\boldsymbol{w}_{r}$ and collisions of like $(s=r)$ and unlike $(s \neq r)$ particles, where $^{\mathbf{1 4}}$

$$
C_{r s}=\int \cdots \int\left[f_{r}^{*} f_{s}^{*}-f_{r} f_{s}\right] g_{r s} \sigma_{r s} \mathrm{~d} \boldsymbol{\Omega} \mathrm{d} \boldsymbol{v}_{s}
$$

are the partial collision integrals of the $(r-s)$-interactions. The initial $\left\{\boldsymbol{v}_{r}, \boldsymbol{v}_{s}\right\}$ and final $\left\{\boldsymbol{v}_{r}^{*}, \boldsymbol{v}_{s}^{*}\right\}$ state in the collisional transition are related by the conservation equations for momentum and energy $(\boldsymbol{e}=$ apseline unit vector) ${ }^{14}$ :

$$
\begin{aligned}
& \boldsymbol{v}_{r}^{*}=\boldsymbol{v}_{\mathrm{r}}+2 \frac{m_{r s}}{m_{r}}\left(\boldsymbol{g}_{r s} \cdot \boldsymbol{e}\right) \boldsymbol{e}, \\
& \boldsymbol{v}_{s}^{*}=\boldsymbol{v}_{s}-2 \frac{m_{s r}}{m_{s}}\left(\boldsymbol{g}_{r s} \cdot \boldsymbol{e}\right) \boldsymbol{e},
\end{aligned}
$$

where

$\boldsymbol{g}_{r s}=\boldsymbol{v}_{s}-\boldsymbol{v}_{r},\left|\boldsymbol{g}_{r s}\right|=\left|\boldsymbol{g}_{r s}^{*}\right|, \boldsymbol{g}_{r s}^{*}=\boldsymbol{v}_{s}^{*}-\boldsymbol{v}_{r}^{*}$.

14 L. Waldmann, Transport Phenomena in Gases of Medium Pressures, Encyclopedia of Physics, Vol. XII, SpringerVerlag, New York 1958.
By specializing to quasi-homogeneous plasmas, $\pi_{r, i j} \rightarrow 0$ and $q_{r, i} \rightarrow 0$, the nonequilibrium distribution function for any component $(r)$ reduces to the displaced Maxwellian ${ }^{1-3}$,

$f_{r}=n_{r}\left(\frac{m_{r}}{2 \pi k T_{r}}\right)^{3 / 2} \exp \left\{-m_{r}\left(\boldsymbol{v}_{r}-\left\langle\boldsymbol{v}_{r}\right\rangle\right)^{2} / 2 k T_{r}\right\}$,

which represents a five-moment-approximation characterized by the individual density $n_{r}$, temperature $T_{r}$, and mean mass velocity $\left\langle\boldsymbol{v}_{r}\right\rangle$ of the $r$-particles.

The elastic collisions of the charged particles $\left(e_{r}\right)$ and $\left(e_{s}\right)$, which interact by a Coulomb potential shielded beyond the Debye radius, are described by the differential cross section ${ }^{14}$

$$
\begin{aligned}
\sigma_{r s} & =\frac{1}{4}\left(\frac{e_{r} e_{s}}{m_{r s} g_{r s}^{2}}\right)^{2} \sin ^{-4}(\varkappa / 2), & & \text { for : } \varkappa_{\min } \leqq \varkappa \leqq \pi, \\
& =0 & & 0 \leqq \varkappa \leqq \varkappa_{\min },
\end{aligned}
$$

referred to the center of mass system. The minimum scattering angle $\varkappa_{\min }\left[\varkappa=\Varangle\left(\boldsymbol{g}_{r s}, \boldsymbol{g}_{r s}^{*}\right)\right]$, which corresponds to the maximum impact parameter $\varrho_{\max }=D$, is

where

$$
\varkappa_{\min }=2 \operatorname{arc} \operatorname{ctg}\left(D / \varrho_{\perp}\right),
$$

and

$$
D=\left(4 \pi \sum_{s} n_{s} e_{s}^{2} / k T_{s}\right)^{-1 / z}
$$$$
\varrho \perp=\left|e_{r} e_{s}\right| /\left(m_{r s} g_{r s}^{2}\right) .
$$

\section{Mathematical Preliminaries}

Transformations. - Because of the invariance of the Eqs. (3) and (4) with respect to Galilei transformation, similar equations hold also for the thermal velocities $\boldsymbol{c}_{r}^{*}$ and $\boldsymbol{c}_{s}^{*}$ :

$$
\begin{gathered}
\boldsymbol{c}_{r}^{*}=\boldsymbol{c}_{r}+2 \frac{m_{r s}}{m_{r}}\left(\boldsymbol{g}_{r s} \cdot \boldsymbol{e}\right) \boldsymbol{e}, \\
\boldsymbol{c}_{s}^{*}=\boldsymbol{c}_{s}-2 \frac{m_{s r}}{m_{s}}\left(\boldsymbol{g}_{r s} \cdot \boldsymbol{e}\right) \boldsymbol{e},
\end{gathered}
$$

where

$$
\boldsymbol{c}_{r}=\boldsymbol{v}_{r}-\left\langle\boldsymbol{v}_{r}\right\rangle, \ldots, \boldsymbol{c}_{s}^{*}=\boldsymbol{v}_{s}^{*}-\left\langle\boldsymbol{v}_{s}^{*}\right\rangle .
$$

In terms of the variables,

and

$$
\boldsymbol{c}_{r s}=\left[a_{r} /\left(a_{r}+a_{s}\right)\right] \boldsymbol{c}_{r}+\left[a_{s} /\left(a_{s}+a_{r}\right)\right] \boldsymbol{c}_{s}
$$

$$
\boldsymbol{g}_{r s}\left(\boldsymbol{c}_{r s}=\boldsymbol{c}_{s r}, \boldsymbol{g}_{r s}=-\boldsymbol{g}_{s r}\right),
$$

the thermal velocities become:

$$
\begin{aligned}
& \boldsymbol{c}_{r}=\boldsymbol{c}_{r s}-\frac{a_{r s}}{a_{r}}\left(\boldsymbol{g}_{r s}-\left\langle\boldsymbol{g}_{r s}\right\rangle\right), \\
& \boldsymbol{c}_{s}=\boldsymbol{c}_{r s}+\frac{\boldsymbol{a}_{s r}}{\boldsymbol{a}_{s}}\left(\boldsymbol{g}_{r s}-\left\langle\boldsymbol{g}_{r s}\right\rangle\right),
\end{aligned}
$$


where

$$
a_{r}=\frac{m_{r}}{2 k T_{r}}, \quad a_{r s}=\frac{a_{r} a_{s}}{a_{r}+a_{s}}, \quad a_{s}=\frac{m_{s}}{2 k T_{s}} .
$$

Since the Jacobians are $\left|\left[\partial\left(\boldsymbol{v}_{r}, \boldsymbol{v}_{s}\right) / \partial\left(\boldsymbol{c}_{r}, \boldsymbol{c}_{s}\right)\right]\right|=1$ and $\left|\left[\partial\left(\boldsymbol{c}_{r}, \boldsymbol{c}_{s}\right) / \partial\left(\boldsymbol{c}_{r s}, \boldsymbol{g}_{r s}\right)\right]\right|=1$, the products of the velocity space elements in the different variables equal,

$$
\mathrm{d} \boldsymbol{v}_{r} \mathrm{~d} \boldsymbol{v}_{s}=\mathrm{d} \boldsymbol{c}_{r} \mathrm{~d} \boldsymbol{c}_{s}=\mathrm{d} \boldsymbol{c}_{r s} \mathrm{~d} \boldsymbol{g}_{r s} .
$$

As an illustration of the transformation, Eqs. (14) and (15), consider the partial integration $\mathrm{d} \boldsymbol{c}_{r s}$ of the integration $\mathrm{d} \boldsymbol{v}_{r} \mathrm{~d} \boldsymbol{v}_{s}=\mathrm{d} \boldsymbol{c}_{r s} \mathrm{~d} \boldsymbol{g}_{r s}$ over $f_{r} f_{s}$ :

$$
\begin{aligned}
f_{r s}=\iint_{-\infty}^{+\infty} \int_{r} f_{r} f_{s} \mathrm{~d} \boldsymbol{c}_{r s}=n_{r} n_{s}\left(\frac{a_{r s}}{\pi}\right)^{3 / 2} \\
\cdot \exp \left\{-a_{r s}\left(\boldsymbol{g}_{r s}-\left\langle\boldsymbol{g}_{r s}\right\rangle\right)^{2}\right\} .
\end{aligned}
$$

Expansions. - A series development of Eq. (18) up to terms of second order in $\sqrt{a_{r s}}\left|\left\langle\boldsymbol{g}_{r s}\right\rangle\right|$ yields for the infrasonic limit, $\sqrt{a_{r s}}\left|\left\langle\boldsymbol{g}_{r s}\right\rangle\right| \ll 1$ :

$$
\begin{aligned}
f_{r s}=n_{r} n_{s} & \left(\frac{a_{r s}}{\pi}\right)^{3 / 2} \exp \left\{-a_{r s} \boldsymbol{g}_{r s}^{2}\right\} \\
& {\left[1+2 \sqrt{a_{r s}} \boldsymbol{g}_{r s} \cdot \sqrt{a_{r s}}\left\langle\boldsymbol{g}_{r s}\right\rangle-\left(\sqrt{a_{r s}}\left\langle\boldsymbol{g}_{r s}\right\rangle\right)^{2}\right.} \\
+ & \left.2\left(\sqrt{a_{r s}} \boldsymbol{g}_{r s} \cdot \sqrt{a_{r s}}\left\langle\boldsymbol{g}_{r s}\right\rangle\right)^{2}-\ldots\right] .
\end{aligned}
$$

The analytical considerations lead among other things to the Maxwell integral $\mu\left(x^{2}\right)$ and the error function $\Phi(x)$, which are related by

$\mu\left(x^{2}\right)=\Phi(x)-\frac{2}{\sqrt{\pi}} x e^{-x^{2}}, \quad \Phi(x) \equiv \frac{2}{\sqrt{\pi}} \int_{0}^{x} e^{-x^{\prime 2}} \mathrm{~d} x^{\prime}$.

$\mu\left(x^{2}\right)$ and $\Phi(x)$ have the series expansions ${ }^{15}$ :

$$
\begin{aligned}
\mu\left(x^{2}\right)= & 1-\frac{2}{\sqrt{\pi}} x e^{-x^{2}} \\
& \cdot\left[1+\sum_{m=0}^{\infty}(-1)^{m} \frac{(2 m-1) ! !}{2^{m+1} x^{2(m+1)}}\right] \text { for }: x>1
\end{aligned}
$$$$
=\frac{4}{\sqrt{\pi}} \sum_{m=0}^{\infty}(-1)^{m+1} \frac{x^{2 m+1}}{(2 m+1)(m-1) !}
$$

and

$$
\text { for: } x \lessgtr 1
$$

$$
\begin{aligned}
& \Phi(x)=1-\frac{2}{\sqrt{\pi}} x e^{-x^{2}} \sum_{m=0}^{\infty}(-1)^{m} \frac{(2 m-1) ! !}{2^{m+1} x^{2(m+1)}} \\
& \text { for: } x>1 \\
& =\frac{2}{\sqrt{\pi}} \sum_{m=0}^{\infty}(-1)^{m} \frac{x^{2 m+1}}{(2 m+1) m !} \text { for : } x \lessgtr 1 \text {. }
\end{aligned}
$$

15 M. Abramowitz and I. A. Stegun, Handbook of Mathematical Functions, Dover Publ., Inc., New York 1965.

\section{Intercomponent Momentum Transfer}

A component $(r)$ may be pictured as a beam of $r$-particles of macroscopic velocity $\left\langle\boldsymbol{v}_{r}\right\rangle$ with superimposed thermal motions $\boldsymbol{c}_{r}$ at the temperature $T_{r}$. Due to collisions of $r$-particles with $s$-particles, momentum is exchanged between the $r$ - and $s$-beams. According to Eq. (1), the force density exerted by the $s$-component on the $r$-component is the collision integral with respect to the dynamical variable $m_{r} \boldsymbol{v}_{r}$ :

$$
\boldsymbol{F}_{r s}=m_{r} \int \cdots \int \boldsymbol{v}_{r}\left[f_{r}^{*} f_{s}^{*}-f_{r} f_{s}\right] g_{r s} \sigma_{r s} \mathrm{~d} \boldsymbol{\Omega} \mathrm{d} \boldsymbol{v}_{r} \mathrm{~d} \boldsymbol{v}_{s} .
$$

A symmetrical transformation ${ }^{14}$, and substitution for $\left(\boldsymbol{v}_{r}^{*}-\boldsymbol{v}_{r}\right)$ in accordance with Eq. (3) gives

$\boldsymbol{F}_{r s}=2 m_{r s} \int \cdots \int\left(\boldsymbol{g}_{r s} \cdot \boldsymbol{e}\right) \boldsymbol{e} f_{r} f_{s} g_{r \mathrm{~s}} \sigma_{r s} \mathrm{~d} \boldsymbol{\Omega} \mathrm{d} \boldsymbol{v}_{r} \mathrm{~d} \boldsymbol{v}_{s}$.

Introduce a spherical coordinate system $(1, \Theta, \Phi)$ with the polar axis parallel to $\boldsymbol{g}_{r s}$, in which

$$
\begin{aligned}
\boldsymbol{e} & =\{\sin \Theta \cos \Phi, \sin \Theta \sin \Phi, \cos \Theta\}, \\
\mathrm{d} \boldsymbol{\Omega} & =-4 \sin \Theta \cos \Theta \mathrm{d} \Theta \mathrm{d} \Phi .,
\end{aligned}
$$

The partial integral of Eq. (23) with respect to $d \Phi$ is

$\int_{0}^{2 \pi} \boldsymbol{e} \boldsymbol{e} \mathrm{d} \Phi=\pi\left[\sin ^{2} \Theta \overleftrightarrow{\boldsymbol{\delta}}+\left(2 \cos ^{2} \Theta-\sin ^{2} \Theta\right) \frac{\boldsymbol{g}_{r s} \boldsymbol{g}_{r s}}{g_{r s}^{2}}\right]$

Under consideration of Eqs. (17) and (24), Eq. (23) becomes

$$
\boldsymbol{F}_{r s}=4 \pi m_{r s} \int \cdots \int \boldsymbol{g}_{r s} g_{r s} \Lambda\left(g_{r s}\right) f_{r} f_{s} \mathrm{~d} \boldsymbol{c}_{r s} \mathrm{~d} \boldsymbol{g}_{r s},
$$

where

$$
A\left(g_{r s}\right)=-4 \pi \int_{\Theta_{\min }}^{\Theta_{\max }} \sigma_{r s}\left(g_{r s}, \Theta\right) \cos ^{3} \Theta \sin \Theta \mathrm{d} \Theta,
$$

whence,

$\Lambda\left(g_{r s}\right)=\frac{1}{2}\left(\frac{e_{r} e_{s}}{m_{r s}}\right)^{2} g_{r s}^{-4} \ln \left[1+\left(\frac{D m_{r s}}{e_{r} e_{s}}\right)^{2} g_{r s}^{4}\right]$

for the Coulomb cross section, Eq. (7), and $\Theta_{\max }=\pi$ and $\cos ^{2} \Theta_{\min }=\left[1+\left(D / \varrho_{\perp}\right)^{2}\right]^{-1}$, Eqs. $(8-10)$. Combining Eqs. (25) and (26), and integrating with respect to d $\boldsymbol{c}_{r s}$, Eq. (18), yields

$$
\boldsymbol{F}_{r s}=2 \pi m_{r s} n_{r} n_{s}\left(\frac{a_{r s}}{\pi}\right)^{3 / 2}\left(\frac{e_{r} e_{s}}{m_{r s}}\right)^{2} \frac{\left\langle\boldsymbol{g}_{r s}\right\rangle}{\left|\left\langle\boldsymbol{g}_{r s}\right\rangle\right|} \mathcal{G},
$$

where

$$
\begin{aligned}
\mathcal{G}= & \iiint_{-\infty}^{+\infty} \exp \left\{-\boldsymbol{a}_{r s}\left(\boldsymbol{g}_{r s}-\left\langle\boldsymbol{g}_{r s}\right\rangle\right)^{2}\right\} \\
& \cdot \ln \left[1+\left(\frac{D m_{r s}}{e_{r} e_{s}}\right)^{2} g_{r s}^{4} \mid \frac{\left\langle\boldsymbol{g}_{r s}\right\rangle \cdot \boldsymbol{g}_{r s}}{\left|\left\langle\boldsymbol{g}_{r s}\right\rangle\right| g_{r s}^{3}} \mathrm{~d} \boldsymbol{g}_{r s} .\right.
\end{aligned}
$$


Introduce a spherical coordinate system $\left(g_{r s}, \alpha, \beta\right)$ with the polar axis paralled to $\left\langle\boldsymbol{g}_{r s}\right\rangle$, in which

$$
\boldsymbol{g}_{r s}=g_{r s}\{\sin \alpha \cos \beta, \sin \alpha \sin \beta, \cos \alpha\},
$$

$$
\text { B: } \quad \alpha=\Varangle\left(\boldsymbol{g}_{r s},\left\langle\boldsymbol{g}_{r s}\right\rangle\right),
$$$$
\mathrm{d} \boldsymbol{g}_{r s}=g_{s r}^{2} \mathrm{~d} g_{r s} \sin \alpha \mathrm{d} \alpha \mathrm{d} \beta,
$$

and the substitution,

$$
\begin{aligned}
& \cos \boldsymbol{\alpha}=\tau, \quad \mathrm{d} \tau=-\sin \alpha \mathrm{d} \alpha, \\
& \mathrm{C}: \quad, \quad \gamma_{r s}=\boldsymbol{a}_{r s}^{1 / 2}\left|\left\langle\boldsymbol{g}_{r s}\right\rangle\right| . \\
& \boldsymbol{a}_{r s}^{1 / 2} g_{r s}=x, \quad \mathrm{~d} x=\boldsymbol{a}_{r s}^{1 / 2} \mathrm{~d} g_{r s}
\end{aligned}
$$

The operations (B) and (C) transform the integral in Eq. (28) to

$$
\begin{aligned}
& \mathcal{G}=2 \pi a_{r s}^{-1 / 2} \int_{\tau=-1}^{+1} \int_{x=0}^{\infty} \tau e^{-\left(x^{2}+\gamma_{r s}^{2}-2 \gamma_{r s} x \tau\right)} \\
& \cdot \ln \left[1+\left(\frac{D m_{r s}}{e_{r} e_{s} a_{r s}}\right)^{2} x^{4}\right] \mathrm{d} x \mathrm{~d} \tau,
\end{aligned}
$$

whence,

$$
\begin{aligned}
& G=2 \pi a_{r s}^{-1 / 2} \sum_{\sigma= \pm 1} \int_{x=0}^{\infty} \frac{2 \gamma_{r s} x-\sigma}{\left(2 \gamma_{r s} x\right)^{2}} e^{-\left(x-\gamma_{r s} \sigma\right)^{2}} \\
& \cdot \ln \left[1+\left(\frac{D m_{r s}}{e_{r} e_{s} a_{r s}}\right)^{2} x^{4}\right] \mathrm{d} x .
\end{aligned}
$$

[Note that the sum (!) of the $\sigma$-integrals, $\sigma= \pm 1$, exists for $x=0$.]

a) Supersonic Drift Velocities,

$$
\gamma_{r s}=\sqrt{\boldsymbol{a}_{r s}}\left|\left\langle\boldsymbol{g}_{r s}\right\rangle\right|>1 \text { : }
$$

The integrals in Eq. (30) have saddlepoints at $x=\gamma_{r s} \sigma, \sigma= \pm 1$, respectively. Since the line of steepest descent through $z=\left(\gamma_{r s} \sigma, 0\right)$ is identical with the real axis, the integration is to be performed along the real coordinate ${ }^{16}$. Thus, one finds in the first approximation for supersonic drift velocities ${ }^{17}$, $\gamma_{r s}>1$ :

$$
\begin{aligned}
& G=2 \pi a_{r s}^{-1 / 2} \lim _{\varepsilon \rightarrow 0}\left\{\sum_{\sigma= \pm 1} \ln \left[1+\left(\frac{D m_{r s}}{e_{r} e_{s} a_{r s}}\right)^{2} x^{4}\right]_{x=\gamma_{r s} \sigma}\right. \\
&\left.\cdot \int_{x=\varepsilon}^{\infty} \frac{2 \gamma_{r s} x-\sigma}{\left(2 \gamma_{r s} x\right)^{2}} e^{-\left(x-\gamma_{r s} \sigma\right)^{2}} \mathrm{~d} x\right\},
\end{aligned}
$$

whence,

$$
\begin{aligned}
\mathcal{G}=\pi\left(\frac{\pi}{a_{r s}}\right)^{1 / 2} \cdot \ln [1 & \left.+\left(\frac{D m_{r s}}{e_{r} e_{s} a_{r s}}\right)^{2} \gamma_{r s}^{4}\right] \\
& \gamma_{r s}^{-2}\left[\Phi\left(\gamma_{r s}\right)-\frac{2}{\sqrt{\pi}} \gamma_{r s} e^{-\gamma_{r s}^{2}}\right] .
\end{aligned}
$$

Substitution of Eq. (31) into Eq. (27) results in the following expression for the force density exert-

16 P. Debye, Math. Ann. 67, 535 [1909].

17 The higher approximations can be shown to be sufficiently small for $\gamma_{r s}>1$, and completely negligible for $\gamma_{r s} \gg 1$. ed by the $s$-component on the $r$-component, $\gamma_{r s}>1$ :

$\boldsymbol{F}_{r s}=-M\left(a_{r s}\left\langle\boldsymbol{g}_{r s}\right\rangle^{2}\right) \tau_{r s}^{-1} n_{r} m_{r}\left(\left\langle\boldsymbol{v}_{r}\right\rangle-\left\langle\boldsymbol{v}_{s}\right\rangle\right)$

where,

$$
\begin{aligned}
M\left(\gamma_{r s}^{2}\right) & =\frac{3 \sqrt{\pi}}{4} \gamma_{r s}^{-3} \mu\left(\gamma_{r s}^{2}\right), \\
\tau_{r s}^{-1} & =\frac{8}{3} \sqrt{\frac{2 k T_{r s}}{\pi m_{r s}}} \frac{m_{r s}}{m_{r}} n_{s} Q_{r s} \neq \tau_{s r}^{-1}, \\
Q_{r s} & =\frac{\pi}{2}\left(\frac{e_{r} e_{s}}{k T_{r s}}\right)^{2} L_{r s}=Q_{s r}, \\
L_{r s} & =\frac{1}{2} \ln \left[1+\left(\frac{D}{e_{r} e_{s}} m_{r s}\left\langle\boldsymbol{g}_{r s}\right\rangle^{2}\right)^{2}\right]=L_{s r} .
\end{aligned}
$$

The force density $\boldsymbol{F}_{r s}$, Eq. (32), is a transcendental function of $\left(\left\langle\boldsymbol{v}_{s}\right\rangle-\left\langle\boldsymbol{v}_{r}\right\rangle\right) . M\left(\gamma_{r s}^{2}\right)$ has the properties,

and

$$
\begin{array}{rlrl}
M\left(\gamma_{r s}^{2}\right) \cong(3 \sqrt{\pi} / 4) & \gamma_{r s}^{-3} & \gamma_{r s} & \gg 1 \\
M\left(\gamma_{r s}^{2}\right) \cong 1 & \text { for : } & \\
\gamma_{r s} & \ll 1
\end{array}
$$

according to Eq. (21). The time characteristic for the build-up or relaxation of the intercomponent force interaction is,

$$
\tau_{r s}^{\mathrm{F}}=\tau_{r s} / M\left(\gamma_{r s}^{2}\right) \neq \tau_{s r}^{\mathrm{F}} .
$$

b) Infrasonic Drift Velocities,

$$
\gamma_{r s}=\sqrt{a_{r s}}\left|\left\langle\boldsymbol{g}_{r s}\right\rangle\right| \ll 1:
$$

An expansion of the $\mathcal{G}$-integral, Eq. (29), for infrasonic drift velocities in accordance with Eq. (19) yields

$$
\begin{aligned}
\mathcal{G}^{*} & =2 \pi a_{r s}^{-1 / 2} \int_{\tau=-1}^{+1} \int_{x=0}^{\infty} \tau e^{-x^{2}}\left[1+2 \gamma_{r s} x \tau-\gamma_{r s}^{2}\right. \\
& \left.+2 \gamma_{r s}^{2} x^{2} \tau^{2}-\ldots\right] \cdot \ln \left[1+\left(\frac{D m_{r s}}{e_{r} e_{s} a_{r s}}\right)^{2} x^{4}\right] \mathrm{d} x \mathrm{~d} \tau .
\end{aligned}
$$

Since only terms proportional to even powers of $\tau$ contribute to the integral, there follows by disregarding terms of second and higher order in $\gamma_{r s}$,

where

$$
G^{*}=(8 \pi / 3) a_{r s}^{-1 / 2} \gamma_{r s} L_{r s}^{*},
$$

$$
L_{r s}^{*}=\frac{1}{2} \int_{x=0}^{\infty} e^{-x^{2}} \ln \left[1+\left(\frac{D m_{r s}}{e_{r} e_{s} a_{r s}}\right)^{2} x^{4}\right] \mathrm{d}\left(x^{2}\right)
$$

The integral over the Coulomb logarithm has the analytical solution ${ }^{18}$

18 D. Bierens De HaAn, Nouvelles Tables D'Intégrales Définies, G. E. Stechert \& Co., New York 1939. 


$$
\begin{array}{r}
L_{r s}^{*}=\frac{\pi}{2} \sin q-[\mathrm{Ci}(q) \cos q+\operatorname{Si}(q) \sin q], \\
q \equiv \frac{\left|e_{r} e_{s}\right| a_{r s},}{D m_{r s}},
\end{array}
$$

where $\operatorname{Ci}(x)$ is the cosine integral and $\mathrm{Si}(x)$ is the sine integral $(\Gamma=0.577 \ldots=\text { Euler's constant })^{15}$,

$$
\begin{aligned}
\mathrm{Ci}(x) & =\Gamma+\ln x+\sum_{m=1}^{\infty}(-1)^{m} \frac{x^{2 m}}{2 m(2 m) !}, \\
\mathrm{Si}(x) & =\sum_{m=0}^{\infty}(-1)^{m} \frac{x^{2 m+1}}{(2 m+1)(2 m+1) !} .
\end{aligned}
$$

Substitution of Eqs. (38) and (40) into Eq. (27) results in the following expression for the force density exerted by the $s$-component on the $r$-component, $\gamma_{r s} \ll 1$ :

$$
\boldsymbol{F}_{r s}=-\tau_{r s}^{*-1} n_{r} m_{r}\left(\left\langle\boldsymbol{v}_{r}\right\rangle-\left\langle\boldsymbol{v}_{s}\right\rangle\right),
$$

where

$$
\begin{aligned}
\tau_{r s}^{*-1} & =\frac{8}{3} \sqrt{\frac{2 k T_{r s}}{\pi m_{r s}}} \frac{m_{r s}}{m_{r}} n_{s} Q_{r s}^{*} \neq \tau_{s r}^{*-1}, \\
Q_{r s}^{*} & =\frac{\pi}{2}\left(\frac{e_{r} e_{s}}{k T_{r s}}\right)^{2} L_{r s}^{*}=Q_{s r}^{*}, \\
L_{r s}^{*} & \cong \ln \left(\frac{D}{\left|e_{r} e_{s}\right|} 2 k T_{r s}\right)-\Gamma \cong L_{s r}^{*} .
\end{aligned}
$$

In the infrasonic case, $\boldsymbol{F}_{r s}$ is proportional to $\left(\left\langle\boldsymbol{v}_{s}\right\rangle-\left\langle\boldsymbol{v}_{r}\right\rangle\right)$. Eq. (45) is an approximation derived from Eq. (40) by noting that the electrostatic energy at the Debye-radius is generally small compared to the thermal energy,

$$
\left|e_{r} e_{s}\right| / D \ll 2 k T_{r s} .
$$

The time characteristic for the build-up or relaxation of this intercompoent force interaction is

$$
\tau_{r s}^{* \mathrm{~F}}=\tau_{r s}^{*} \neq \tau_{s r}^{* \mathrm{~F}} .
$$

The friction forces $\boldsymbol{F}_{r s}$ and $\boldsymbol{F}_{s r}$ [Eq. (32) or (42) ] exerted by the $s$ - on the $r$-component and the $r$ - on the $s$-component, respectively, balance at any time $($ action $=$ reaction $)$,

$$
\boldsymbol{F}_{r s}=-\boldsymbol{F}_{s r} .
$$

\section{Intercomponent Energy Transfer}

In the interpenetrating components $(r)$ and $(s)$ heat is liberated through collisions raising the thermal energy of the components. The energy exchanged in the $(r-s)$-collisions results in a scalar heat flow between the components $(r)$ and $(s)$ of differ- ent temperatures. According to Eq. (1), the energy exchange due to these processes in the $r$-component is given by the collision integral with respect to the dynamical variable $\frac{1}{2} m_{r} c_{r}^{2}$ :

$W_{r s}=\frac{1}{2} m_{r} \int \cdots \int c_{r}^{2}\left[f_{r}^{*} f_{s}^{*}-f_{r} f_{s}\right] g_{r s} \sigma_{r s} \mathrm{~d} \boldsymbol{\Omega} \mathrm{d} \boldsymbol{v}_{r} \mathrm{~d} \boldsymbol{v}_{s}$.

A symmetrical transformation ${ }^{14}$, and substitution for $\left(c_{r}^{* 2}-c_{r}^{2}\right)$ in accordance with Eq. (11) gives

$$
\begin{aligned}
& W_{r s}=2 m_{r s} \int \cdots \int {\left[\frac{m_{r s}}{m_{r}}\left(\boldsymbol{g}_{r s} \cdot \boldsymbol{e}\right)^{2}+\left(\boldsymbol{g}_{r s} \cdot \boldsymbol{e}\right)\right.} \\
&\left.\cdot\left(\boldsymbol{c}_{r} \cdot \boldsymbol{e}\right)\right] f_{r} f_{s} g_{r s} \sigma_{r s} \mathrm{~d} \boldsymbol{\Omega} \mathrm{d} \boldsymbol{v}_{r} \mathrm{~d} \boldsymbol{v}_{s} .
\end{aligned}
$$

The substitution (A), and integration with respect to $\mathrm{d} \Theta$ and $\mathrm{d} \Phi$ yields under consideration of Eqs. (17) and (24)

$$
\begin{aligned}
W_{r s}=4 \pi m_{r s} \int \cdots \int\left[\frac{m_{r s}}{m_{r}}\right. & \left.g_{r s}^{2}+\boldsymbol{g}_{r s} \cdot \boldsymbol{c}_{r}\right] \\
& \cdot g_{r s} \Lambda\left(g_{r s}\right) f_{r} f_{s} \mathrm{~d} \boldsymbol{c}_{r s} \mathrm{~d} \boldsymbol{g}_{r s},
\end{aligned}
$$

where $A\left(g_{r s}\right)$ is the $\Theta$-integral evaluated in Eq. (26). Substitution for $\boldsymbol{c}_{r}$ in accordance with Eq. (14), and integration with respect to d $\boldsymbol{c}_{r s}$, Eq. (18), yields

$$
\begin{gathered}
W_{r s}=4 \pi m_{r s} n_{r} n_{s}\left(\frac{a_{r s}}{\pi}\right)^{3 / 2} \iiint_{-\infty}^{+\infty} \exp \left\{-\boldsymbol{a}_{r s}\left(\boldsymbol{g}_{r s}-\left\langle\boldsymbol{g}_{r s}\right\rangle\right)^{2}\right\} \\
\cdot\left[\frac{\boldsymbol{a}_{r s}}{\boldsymbol{a}_{r}} \boldsymbol{g}_{r s} \cdot\left\langle\boldsymbol{g}_{r s}\right\rangle+\left(\frac{m_{r s}}{m_{r}}-\frac{a_{r s}}{a_{r}}\right) g_{r s}^{2}\right] g_{r s} \Lambda\left(g_{r s}\right) \mathrm{d} \boldsymbol{g}_{r s} .
\end{gathered}
$$

\section{Intercomponent Heat Generation}

The first integral expression of Eq. (46) represents the power $W_{r s}^{\mathrm{F}}$ liberated by the friction force in the $r$-component. By insertion of $A\left(g_{r s}\right)$, Eq. (26), there obtains

$$
\begin{aligned}
W_{r s}^{\mathrm{F}}= & 2 \pi m_{r s} n_{r} n_{s}\left(\frac{a_{r s}}{\pi}\right)^{3 / 2}\left(\frac{e_{r} e_{s}}{m_{r s}}\right)^{2} \frac{a_{r s}}{a_{r}} \\
& \cdot \iiint_{-\infty}^{+\infty} \exp \left\{-a_{r s}\left(\boldsymbol{g}_{r s}-\left\langle\boldsymbol{g}_{r s}\right\rangle\right)^{2}\right\} \\
& \cdot \ln \left[1+\left(\frac{D m_{r s}}{e_{r} e_{s}}\right)^{2} g_{r s}^{4}\right] \frac{\left\langle\boldsymbol{g}_{r s}\right\rangle \cdot \boldsymbol{g}_{r s}}{g_{r s}^{3}} \mathrm{~d} \boldsymbol{g}_{r s} .
\end{aligned}
$$

The substitutions (B) and (C) transform this equation to,

$$
\left.\left.W_{r s}^{\mathrm{F}}=2 \pi m_{r s} n_{r} n_{s}\left(\frac{a_{r s}}{\pi}\right)^{3 / 2}\right) \frac{e_{r} e_{s}}{m_{r s}}\right)^{2} \frac{a_{r s}}{a_{r}}\left|\left\langle\boldsymbol{g}_{r s}\right\rangle\right| \cdot \mathcal{G},
$$

where $\mathcal{G}$ is the integral defined in Eq. (29). 
a) Supersonic Drift Velocities,

$$
\gamma_{r s}=\sqrt{a_{r s}}\left|\left\langle\boldsymbol{g}_{r s}\right\rangle\right|>1 \text { : }
$$

The $G$-integral, Eq. (29), has been evaluated in Eq. (31) for supersonic drift velocities. By combining Eqs. (31) and (47), the thermal energy liberated per unit volume and time by intercomponent friction in the $r$-component obtains as, $\gamma_{r s}>1$ : $W_{r s}^{\mathrm{F}}=M\left(a_{r s}\left\langle\boldsymbol{g}_{r s}\right\rangle^{2}\right) \frac{T_{r}}{T_{r s}} \tau_{r s}^{-1} n_{r} m_{r s}\left(\left\langle\boldsymbol{v}_{s}\right\rangle-\left\langle\boldsymbol{v}_{r}\right\rangle\right)^{2}$,

where $M\left(\gamma_{\mathrm{v}}^{2}\right)$ and $\tau_{r s}$ are given in Eqs. (33) and (34), respectively. $W_{r s}^{\mathrm{F}}$ is a transcendental function of $\left(\left\langle\boldsymbol{v}_{\mathrm{s}}\right\rangle-\left\langle\boldsymbol{v}_{r}\right\rangle\right)$.

b) Infrasonic Drift Velocities,

$$
\gamma_{r s}=\sqrt{a_{r s}}\left|\left\langle\boldsymbol{g}_{r s}\right\rangle\right| \ll 1:
$$

The $G$-integral, Eq. (29), has been evaluated in Eq. (38) for infrasonic drift velocities. By combining Eqs. (38) and (47), the thermal energy liberated per unit volume and time by intercomponent friction in the $r$-component obtains as, $\gamma_{r s} \ll 1$ :

$$
W_{r s}^{\mathrm{F}}=\frac{T_{r}}{T_{r s}} \tau_{r s}^{*-1} n_{r} m_{r s}\left(\left\langle\boldsymbol{v}_{s}\right\rangle-\left\langle\boldsymbol{v}_{r}\right\rangle\right)^{2},
$$

where $\tau_{r \delta}^{*}$ is given in Eq. (43). In the case $\gamma_{r s} \ll 1$, $W_{r s}^{\mathrm{F}}$ is proportional to $\left(\left\langle\boldsymbol{v}_{s}\right\rangle-\left\langle\boldsymbol{v}_{r}\right\rangle\right)^{2}$.

\section{Intercomponent Heat Flow}

The second integral expression of Eq. (46) represents the scalar heat flow $W_{r s}^{\mathrm{T}}$ from the $r$ - to the $s$-component. By insertion of $\Lambda\left(g_{r s}\right)$, Eq. (26), there obtains

$$
\begin{gathered}
W_{r s}^{\mathrm{T}}=2 \pi m_{r s} n_{r} n_{s}\left(\frac{a_{r s}}{\pi}\right)^{3 / 2}\left(\frac{e_{r} e_{s}}{m_{r s}}\right)^{2}\left(\frac{m_{r s}}{m_{r}}-\frac{a_{r s}}{a_{r}}\right) \\
\cdot \iiint_{-\infty}^{+\infty} \exp \left\{-a_{r s}\left(\boldsymbol{g}_{r s}-\left\langle\boldsymbol{g}_{r s}\right\rangle\right)^{2}\right\} \\
\cdot \ln \left[1+\left(\frac{D m_{r s}}{e_{r} e_{s}}\right)^{2} g_{r s}^{4}\right] \frac{\mathrm{d} \boldsymbol{g}_{r s}}{g_{r s}} .
\end{gathered}
$$

The substitutions (B) and (C) transform this equation to,

$$
W_{r s}^{\mathrm{T}}=2 \pi m_{r s} n_{r} n_{s}\left(\frac{a_{r s}}{\pi}\right)^{3 / 2}\left(\frac{e_{r} e_{s}}{m_{r s}}\right)^{2}\left(\frac{m_{r s}}{m_{r}}-\frac{a_{r s}}{a_{r}}\right) \cdot \mathcal{H},
$$

where

$$
\begin{array}{r}
\mathcal{H}=\frac{2 \pi}{a_{r s}} \int_{\tau=-1}^{+1} \int_{x=0}^{\infty} x e^{-\left(x^{2}+\gamma_{r s}^{2}-2 \gamma_{r s} x \tau\right)} \\
\cdot \ln \left[1+\left(\frac{D m_{r s}}{e_{r} e_{s} a_{r s}}\right)^{2} x^{4}\right] \mathrm{d} x \mathrm{~d} \tau
\end{array}
$$

whence,

$$
\begin{aligned}
\mathcal{H}=\left(2 \pi / a_{r s} \gamma_{r s}\right) & \sum_{\sigma= \pm 1}^{\infty} \sigma \int_{x=0}^{\infty} x e^{-\left(x-\gamma_{r s} \sigma\right)^{2}} \\
& \cdot \ln \left[1+\left(\frac{D m_{r s}}{e_{r} e_{s} a_{r s}}\right)^{2} x^{4}\right] \mathrm{d} x .
\end{aligned}
$$

a) Supersonic Drift Velocities,

$$
\gamma_{r s}=\sqrt{\boldsymbol{a}_{r s}}\left|\left\langle\boldsymbol{g}_{r s}\right\rangle\right|>1 \text { : }
$$

The $\mathcal{H}$-integral, Eq. (52), has saddlespoints at $x=\gamma_{r s} \sigma, \sigma= \pm 1$. By means of the saddlepoint method ${ }^{16}$, one finds in first approximation for supersonic drift velocities ${ }^{17}, \gamma_{r s}>1$ :

$$
\begin{aligned}
\mathcal{H}=\frac{\pi \sqrt{\pi}}{2 a_{r s} \gamma_{r s}} \sum_{\sigma= \pm 1} \sigma \ln \left[1+\left(\frac{D m_{r s}}{e_{r} e_{s} a_{r s}}\right)^{2} x^{4}\right]_{x=\gamma_{r s} \sigma} & \cdot\left[\Phi\left(\gamma_{r s} \sigma\right)+1\right],
\end{aligned}
$$

whence

$$
\mathcal{H}=\frac{\pi \sqrt{\pi}}{a_{r s} \gamma_{r s}} \ln \left[1+\left(\frac{D m_{r s}}{e_{r} e_{s} a_{r s}}\right)^{2} \gamma_{r s}^{4}\right] \boldsymbol{\Phi}\left(\gamma_{r s}\right) .
$$

Under consideration of,

$$
\begin{aligned}
{\left[\left(m_{r s} / m_{r}\right)-\right.} & \left.\left(a_{r s} / a_{r}\right)\right] \\
& =\left(m_{r s} / m_{r}\right)\left(m_{r s} / m_{s}\right)\left(T_{s}-T_{r}\right) / T_{r s},
\end{aligned}
$$

the scalar heat flow from the $r$ - to the $s$-component obtains from Eqs. (50) and (53) as, $\gamma_{r s}>1$ :

$$
W_{r s}^{\mathrm{T}}=-S\left(\sqrt{a_{r s}}\left|\left\langle\boldsymbol{g}_{r s}\right\rangle\right|\right) 3 \frac{m_{r s}}{m_{s}} \tau_{r s}^{-1} n_{r} k\left(T_{r}-T_{s}\right),
$$

where

$$
S\left(\gamma_{r s}\right)=\frac{\sqrt{\pi}}{2} \frac{\Phi\left(\gamma_{r s}\right)}{\gamma_{r s}},
$$

and $\tau_{r s}$ is given in Eq. (34). $W_{r s}^{\mathrm{T}}$ is a transcendental function of $\left(\left\langle\boldsymbol{v}_{s}\right\rangle-\left\langle\boldsymbol{v}_{r}\right\rangle\right) . S\left(\gamma_{r s}\right)$ has the properties,

$S\left(\gamma_{r s}\right) \cong(\sqrt{\pi} / 2) \gamma_{r s}^{-1}, \quad \quad \gamma_{r s} \gg 1$,

and

for:

$S\left(\gamma_{r s}\right) \cong 1 \quad \gamma_{r s} \ll 1$

according to Eq. (22). The relaxation time of the scalar heat flow is,

$$
\tau_{r s}^{\mathrm{T}}=\frac{1}{2} \frac{m_{s}}{m_{r s}} \tau_{r s} / S\left(\gamma_{r s}\right) \neq \tau_{s r}^{\mathrm{T}} .
$$

b) Infrasonic Drift Velocities,

$$
\gamma_{r s}=\sqrt{\boldsymbol{a}_{r s}}\left|\left\langle\boldsymbol{g}_{r s}\right\rangle\right| \ll 1:
$$

An expansion of the $\mathcal{H}$-integral, Eq. (51), for infrasonic drift velocities in accordance with Eq. (19) yields 


$$
\begin{aligned}
\mathcal{H}^{*}= & \frac{2 \pi}{a_{r s}} \int_{\tau=-1}^{+1} \int_{x=0}^{\infty} x e^{-x^{2}}\left[1+2 \gamma_{r s} x \tau-\gamma_{r s}^{2}\right. \\
& \left.+2 \gamma_{r s}^{2} x^{2} \tau^{2}-\ldots\right] \ln \left[1+\left(\frac{D m_{r s}}{e_{r} e_{s} a_{r s}}\right)^{2} x^{4}\right] \mathrm{d} x \mathrm{~d} \tau .
\end{aligned}
$$

Since only terms proportional to even powers of $\tau$ contribute to the integral, there follows by disregarding terms of first and higher order in $\gamma_{r s}$,

$$
\begin{aligned}
\mathcal{H}^{*}=\frac{4 \pi}{a_{r s}} & \frac{1}{2} \int_{x=0}^{\infty} e^{-x^{2}} \\
& \cdot \ln \left[1+\left(\frac{D m_{r s}}{e_{r} e_{s} a_{r s}}\right)^{2} x^{4}\right] \mathrm{d}\left(x^{2}\right)=\frac{4 \pi}{a_{r s}} L_{r s}^{*},
\end{aligned}
$$

where $L_{r s}^{*}$ is the integral evaluated in Eqs. (40) and (45), respectively. Under consideration of

$$
\begin{aligned}
{\left[\left(m_{r s} / m_{r}\right)-\right.} & \left.\left(a_{r s} / a_{r}\right)\right] \\
& =\left(m_{r s} / m_{r}\right)\left(m_{r s} / m_{s}\right)\left(T_{s}-T_{r}\right) / T_{r s},
\end{aligned}
$$

the scalar heat flow from the $r$ - to the $s$-component obtains from Eqs. (50) and (57) as, $\gamma_{r s} \ll 1$ :

$$
W_{r s}^{\mathrm{T}}=-3 \frac{m_{r s}}{m_{s}} \tau_{r s}^{*-1} n_{r} k\left(T_{r}-T_{s}\right),
$$

where $\tau_{r s}^{*}$ is given in Eq. (43). In the case $\gamma_{r s} \ll 1$, $W_{r s}^{\mathrm{T}}$ is independent of $\left(\left\langle\boldsymbol{v}_{s}\right\rangle-\left\langle\boldsymbol{v}_{r}\right\rangle\right)$. The relaxation time of this scalar heat flow is,

$$
\tau_{r s}^{* \mathrm{~T}}=\frac{1}{2} \frac{m_{s}}{m_{r s}} \tau_{r s}^{*} \neq \tau_{s r}^{* \mathrm{~T}} .
$$

The scalar heat flows $W_{r s}^{\mathrm{T}}$ and $W_{g r}^{\mathrm{T}}$ [Eqs. (54) or $(58)]$ from the $r$ - to the $s$-component and the $s$ - to the $r$-component, respectively, balance at any time,

$$
W_{r s}^{\mathrm{T}}=-W_{s r}^{\mathrm{T}} .
$$

\section{Conclusion}

The intercomponent transport processes in nonisothermal plasmas with collisional Coulomb interactions have been evaluated under consideration of the velocity dependence of the Coulomb logarithms for subsonic and supersonic drift velocities of the components. It has been shown that:

\footnotetext{
19 E. Goldman and C. Sirovich, Phys. Fluids 10, 1928 [1967].
}

The transport relations are strongly dependent through transcendental functions on the drift velocities. In particular, completely different functional dependences result in the cases of infrasonic and supersonic drift velocities.

The effective Coulomb logarithms are different for infrasonic and supersonic drift velocities. To the extent that the Coulomb logarithms are large of order 10 , this difference is quantitatively important only in the extreme case of suprasonic drift velocities.

The transport relations obtained for infrasonic drift velocities hold in good approximation for nearly the whole region of subsonic drift velocities, since $M\left(\gamma_{r s}^{2}\right)$ and $S\left(\gamma_{r s}\right)$ do not deviate considerably from 1 for proper subsonic conditions,

$$
1 \geqq M\left(\gamma_{r s}^{2}\right)>\frac{3 \sqrt{\pi}}{4} \mu(1) \cong 0.558
$$

and

$$
\text { for } 0 \leqq \gamma_{r s}<1 \text {. }
$$

$$
1 \geqq S\left(\gamma_{r s}\right)>\frac{\sqrt{\pi}}{2} \Phi(1) \cong 0.747
$$

The assumption of a quasi-homogeneous plasma has no further physical implications. The intercomponent transport processes are hardly influenced by inhomogeneities of ordinary extent in the components for which $\left(\mu_{r}=\right.$ viscosity, $\lambda_{r}=$ conductivity) $\mu_{r}\left|\nabla_{i}\left\langle v_{r, j}\right\rangle\right| \ll p_{r}, \lambda_{r}\left|\nabla_{i} T_{r}\right| \ll p_{r}\left(k T_{r} / m_{r}\right)^{1 / 2}$.

In applications of the transport theory presented it should be noted that (approximate) Maxwellian velocity distributions have been assumed in the c.m.s of the components. Formally, the five-momentapproximation, Eq. (6), folows from the thirteenmoment-approximation and also from the generalized Chapman-Enskog method ${ }^{18}$. In presence of very strong external fields, the hypothesis of a displaced Maxwellian seems to be valid at least as a rough approximation ${ }^{19}$.

\section{Acknowledgement}

The investigation was sponsored by the U.S. Office of Naval Research under the auspices of J. A. SATKOWSKI.

20 H. Dreicer, Phys. Rev. 115, 238 [1959]. 